\title{
Synthesis and Characterization of Click Nucleic Acid Conjugated Polymeric Microparticles for DNA Delivery Applications
}

Alex J. Anderson ${ }^{1}$, Emerson Grey ${ }^{1}$, Nicholas Bongiardina ${ }^{2}$, Christopher N. Bowman ${ }^{1,2,3}$, and Stephanie J. Bryant ${ }^{1,2,3}$,

\section{Supporting Information}

$\underline{\text { Table of contents }}$

Experimental Methods

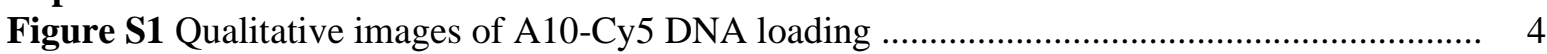

Figure S2 Calibration curves to quantify DNA loading ......................................................... 5

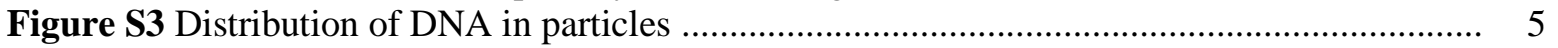

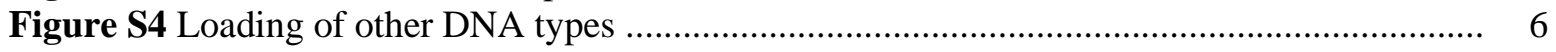

Figure S5 Calibration curves to quantify DNA release ….......................................................... 7

Figure S6 More images of microparticle uptake ....................................................................... 7

Movie S1 3D rendering of microparticle uptake 


\section{Materials.}

Dipentaerythritol hexakis(3-mercaptopropionate) (Di-PETHP) was obtained from Bruno Bock Thiochemicals. Trimethylolpropane triacrylate (TMPTA), polyvinylpyrrolidone (PVP), 4methoxyphenol (MEHQ), and 2,2-dimethyoxy-2-phenylacetophenone (DMPA) were obtained from Sigma Aldrich. Fluorescent DNA strands were obtained from Integrated DNA Technologies. RAW 264.7 macrophages were obtained from ATCC. Phosphate buffered saline (PBS), Dulbecco's Modified Eagle's Medium (DMEM), and penicillin/streptomycin were obtained from Corning. Fetal bovine serum (FBS) was obtained from Atlanta Biologics. Coverslip quality, glass bottom cell culture plates were obtained from Ibidi ( $\mu$ Plates). The cell dyes, Hoechst 33342 and CellTracker Orange, were obtained from ThermoFisher/Invitrogen.

\section{Synthesis of monodisperse, step-growth microparticles.}

Microparticles were synthesized following a previously established protocol. ${ }^{1,2}$ Briefly, a hexafunctional thiol (Di-PETHP) and a triacrylate (TMPTA) were mixed in methanol at a ratio that resulted in a $20 \%$ excess of thiols. To this solution was added the surfactant, PVP, and the radical inhibitor, MEHQ. The solution was mixed thoroughly and the base catalyst (TEA, 5\% by weight with respect to monomers) was added. The mixture was stirred at room temperature for at least 5 hours. The resulting particles were washed extensively with mixtures of acetone and methanol to remove excess monomer, surfactant, inhibitor, and catalyst. The particles were stored at $4^{\circ} \mathrm{C}$ in 50:50 acetone/methanol until needed.

\section{Synthesis of CNA decorated microparticles}

To obtain CNA functionalized microparticles, click nucleic acid monomers were polymerized as described previously, ${ }^{3,4}$ and the thiolated microparticles were included as a dopant in the polymerization. Briefly, CNA monomer was deprotected with 2 equivalents of $\mathrm{NaOH}$, neutralized, and then extracted into dichloromethane (DCM) and dried under vacuum. Separately, thiolated microparticles were suspended in DMF (containing $0.1 \%$ by weight of the photoinitiator (DMPA)) at a concentration of $40 \mathrm{mg}$ particle / $\mathrm{mL}$. This solution was added to the dried CNA monomer to achieve a monomer concentration of $20 \%$ (wt./vol.). The mixture was then sonicated to enhance diffusion of the monomer into the particle and polymerized with $365 \mathrm{~nm}$ UV light at 12-15 $\mathrm{mW} / \mathrm{cm}^{2}$ for 15 minutes at room temperature. The resulting particles were washed at least 3 times with either acetone or DCM to remove the unreacted monomer and oligomer as well as excess photoinitiator.

\section{Characterization of CNA/microparticle copolymerization}

To characterize the morphology of the CNA decorated microparticles, scanning electron microscope (SEM) images were taken. Microparticles suspended in methanol were applied dropwise to a clean silica wafer. Following solvent evaporation, samples were sputter coated with $3 \mathrm{~nm}$ of gold and imaged with a Hitachi SU3500 (SEM). Size measurements were made using ImageJ software and PDI was calculated with the following formula, $P D I=\left(\frac{\sigma}{\bar{x}}\right)^{2}$, where $\sigma$ is the standard deviation and $\bar{x}$ is the average diameter.

To verify $\mathrm{CNA} /$ microparticle conjugation, aliquots of particles were degraded under basic conditions ( $5 \mathrm{M} \mathrm{NaOH}$ for 30 minutes), neutralized, and dried by lyophilization. The degradation products were analyzed by UV-Vis spectroscopy and ${ }^{1} \mathrm{H}$ NMR in DMSO. In addition, the 
molecular weight and degree of polymerization of the CNA oligomers were calculated using gel permeation chromatography (GPC) (TOSOH - HLC8320GPC) with an internal standard of short CNA oligomers.

\section{Preparation of nucleic acid loaded microparticles}

Prior to loading, the Boc group, protecting the thymine imide, was removed by suspending the particles in a mixture of $\mathrm{MeOH}$ and $1 \mathrm{M} \mathrm{HCl}$ for 2-5 minutes. The solution was neutralized and washed twice with water, twice with a water/methanol mixture, and twice with methanol. For cell experiments, particles were sterilized with $70 \%$ ethanol for 24 hours. DNA loaded microparticles were prepared by first obtaining a $2 \mathrm{mg}$ aliquot of CNA functionalized microparticles. $200 \mu \mathrm{L}$ of phosphate buffered saline (PBS) $(10 \mathrm{mM}, \mathrm{pH} 7.5)$ was added to each sample followed by $1 \mu \mathrm{L}$ of 100 mM Cy5 labelled A10 DNA (A10-Cy5). The particles were fully suspended with sonication and mixed overnight on a vortex shaker at room temperature. Samples were then washed 3x with DI $\mathrm{H}_{2} \mathrm{O}$ to remove excess DNA and salt. Microparticles were imaged on a Nikon Spinning Disc Confocal with a $640 \mathrm{~nm}$ excitation laser. To determine the amount of DNA that remained in the microparticles, the particles were degraded in $5 \mathrm{M} \mathrm{NaOH}$, neutralized with $\mathrm{HCl}$, and lyophilized. $300 \mu \mathrm{L}$ of $\mathrm{DI} \mathrm{H}_{2} \mathrm{O}$ was added and each sample was heated to $50^{\circ} \mathrm{C}$ for 15 minutes. After heating, the samples were quickly centrifuged to remove any insoluble components and $200 \mathrm{uL}$ were taken for fluorescence measurements using a Denovix Spectrophotometer/Fluorometer. Molar amounts of A10-Cy5 DNA were obtained using a calibration curve which confirmed a linear relationship between A10-Cy5 DNA and fluorescence in the concentration range examined.

\section{Evaluation of DNA release properties}

DNA loaded microparticles were suspended in PBS and incubated at various temperatures and in various $\mathrm{pH}$ buffers. At prescribed timepoints, $200 \mu \mathrm{L}$ aliquots were taken for fluorescence analysis. The samples were replenished with fresh PBS and the microparticles were resuspended.

\section{Cell Culture and Microparticle Uptake}

A murine macrophage cell line (RAW 264.7) was cultured in Dulbecco's Modified Eagle's Medium (DMEM) media containing $10 \%$ fetal bovine serum (FBS) and 1X penicillin/streptomycin $37^{\circ} \mathrm{C}$ and $5 \% \mathrm{CO}_{2}$, per the recommended protocols. Near confluent cells were collected for sub-culture and plating using a cell scraper. For uptake experiments, cells were plated at a concentration of 12,500 cells/well in $\mu$ Plates with coverslip quality glass bottoms. After 2 days of growth, cells were treated with A10-Cy5 loaded microparticles at a concentration of 100 $\mu \mathrm{g} / \mathrm{mL}$ for 4 hours at room temperature to allow for phagocytosis. Cells were then washed with PBS and fixed with 4\% PFA at room temperature for 5-10 minutes. Cell nuclei were counterstained with Hoechst 33342 and the cytosol with CellTracker Orange. Imaging was performed on a Nikon Spinning Disc Confocal microscope. Cell nuclei were imaged with Dapi filters (405 nm excitation), the cytosol was imaged with TRITC filters (561 nm excitation), and DNA loaded particles were imaged using Cy5 filters (640 nm excitation).

\section{Metabolic Activity and Cell Viability Assays}

The metabolic activity of cells treated with CNA functionalized microparticles was assessed with alamarBlue $^{\mathrm{TM}}$ (Invitrogen) and cytocompatibility was determined by staining cells with calcein AM (Invitrogen). For each assay, RAW 264.7 cells were plated at a density of 20,000 cells/well and allowed to adhere and grow overnight. Cells were then treated with varying concentrations of 
oligo(thymine) (oligo(T)) functionalized microparticles (MP+oT). Samples of wells were left untreated or treated with $1 \%$ Triton-X, which served as controls for the responses of normally proliferative cells and dead cells, respectively. Each assay was then performed after 24 hours. For the alamarBlue ${ }^{\mathrm{TM}}$ assay, the alamarBlue ${ }^{\mathrm{TM}}$ reagent was added to each well to achieve a 1:10 dilution. Absorbance of each well was read at 570 and $600 \mathrm{~nm}$ after 6 hours and the relative metabolic activity with respect to the negative control was calculated using the following formula:

$$
\% \text { Metabolic Activity }=\frac{\varepsilon_{600 \mathrm{~nm}} A_{570 \mathrm{~nm}}^{\text {sample }}-\varepsilon_{570 \mathrm{~nm}} A_{600 \mathrm{~nm}}^{\text {sample }}}{\varepsilon_{600 \mathrm{~nm}} A_{570 \mathrm{~nm}}^{\text {neg control }}-\varepsilon_{570 \mathrm{~nm}} A_{600 \mathrm{~nm}}^{\text {neg }}}
$$

For the cytocompatibility assay, cells were stained with $2 \mu \mathrm{M}$ calcein AM in PBS for 30 minutes. A sample of untreated wells was left unstained to control for background fluorescence. After staining, the fluorescence of each well was read with a 485/535 excitation/emission wavelength pair. The percentage of live cells relative to the control was calculated by the following formula:

$$
\% \text { Live Cells }=\frac{F l_{485}^{\text {sample }}-F l_{485}^{\text {background }}}{F L_{485}^{\text {neg control }}-F l_{485}^{\text {background }}}
$$

\section{Statistical Analysis}

To determine statistical significance, the appropriate test was applied to the data. For histogram analysis, the Kolmogorov-Smirnov test was used $(n=200)$ at the 0.05 confidence level. Differences in means, all of which were determined to be homogenous and follow Gaussian distributions, were calculated by ANOVA with Tukey's Post-Hoc test modification at the 0.05 confidence level.

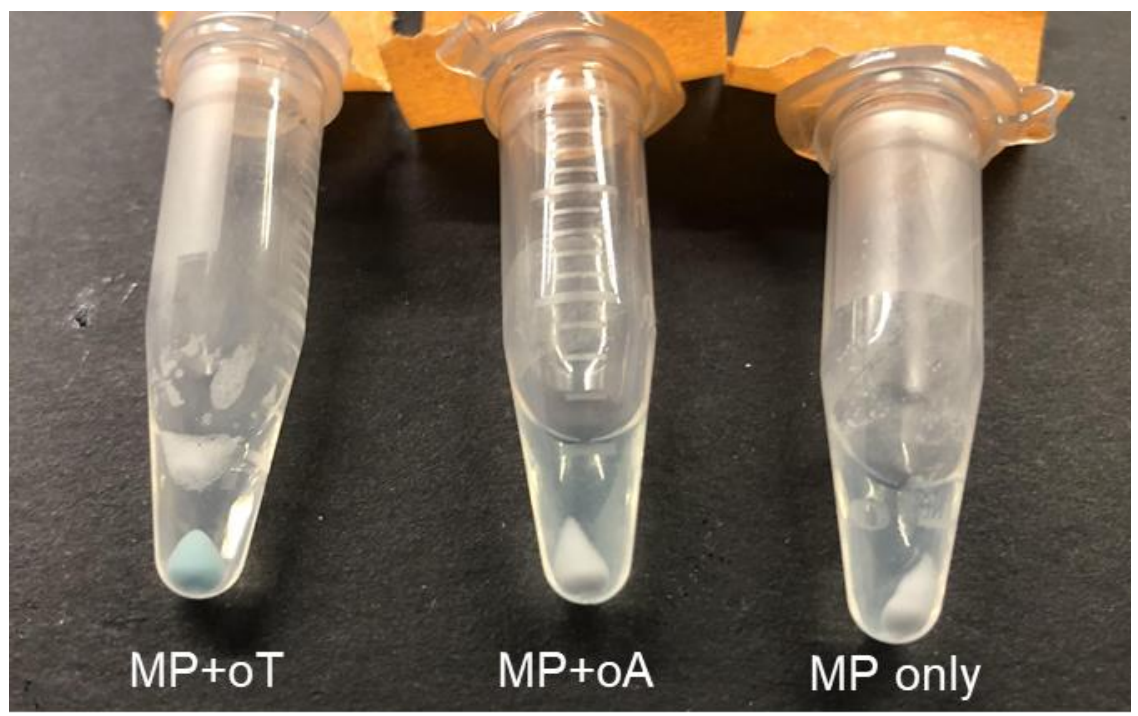

Figure S1-Loading of A10-Cy5 DNA is observed qualitatively by the blue hue of the pellet in the MP+oT samples. The MP+oA and MP only samples did not have the same color. 


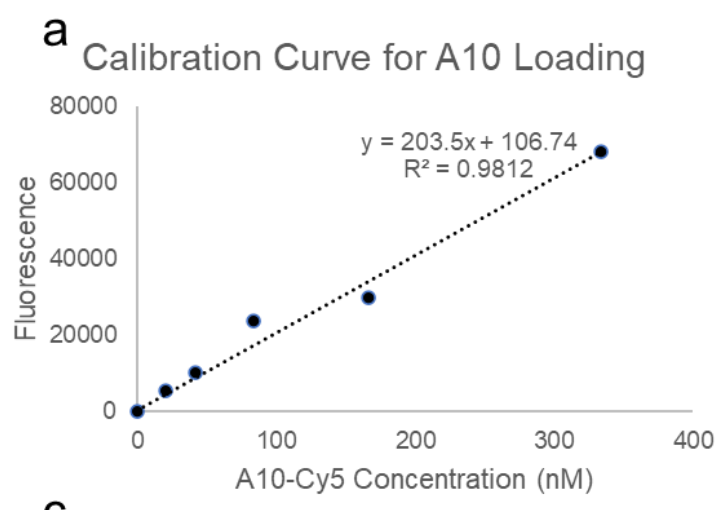

C
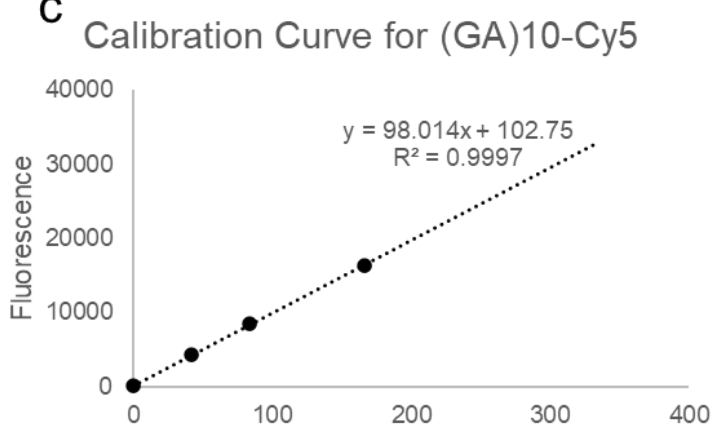

(GA)10-Cy5 Concentration (nM)

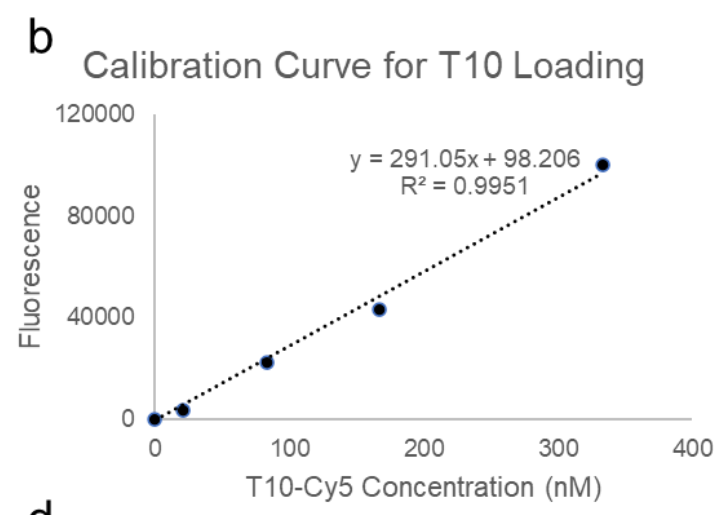

d

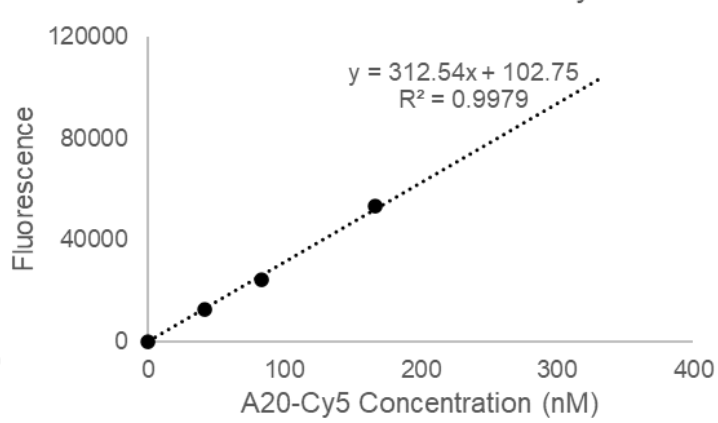

Figure S2 - Calibration curves to quantify the loading of different types of Cy-5 labelled sSDNA: (a) A10Cy5, (b) T10-Cy5, (c) (GA)10-Cy5, and (d) A20-Cy5.
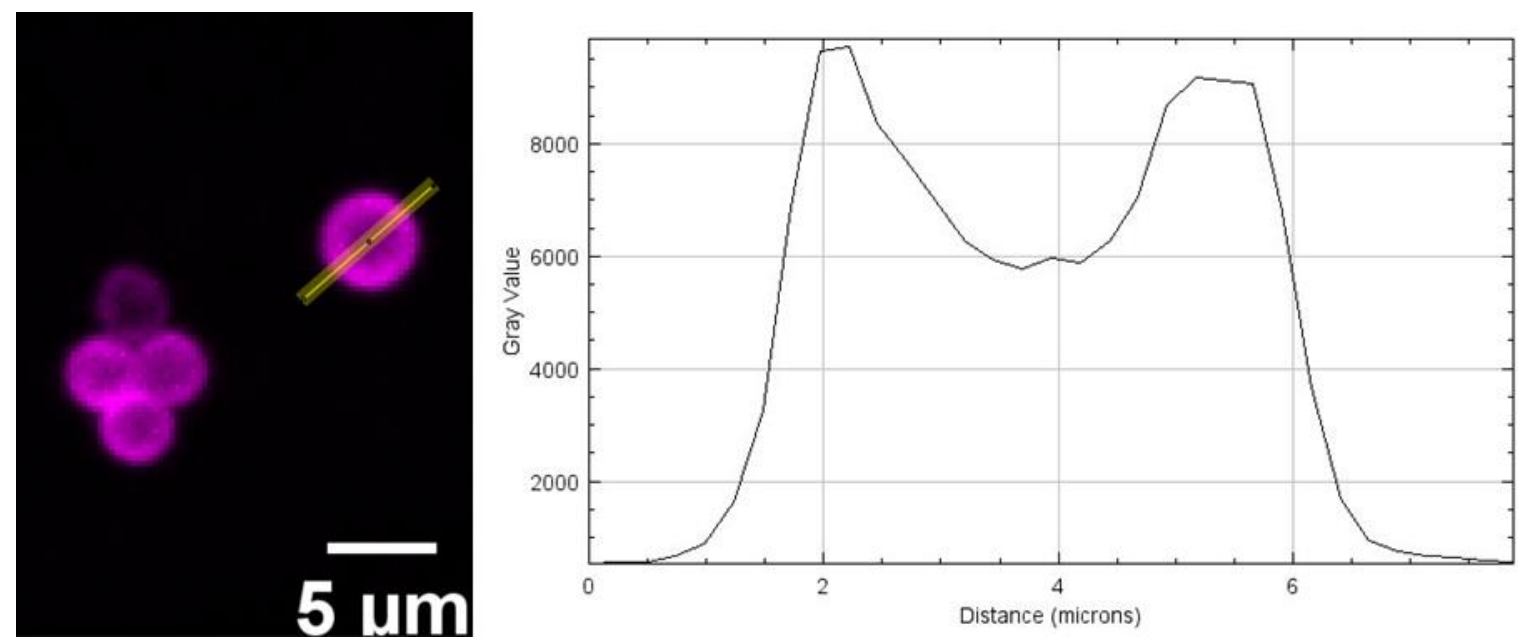

Figure S3 - Confocal microscopy of A10-Cy5 loaded particles reveal a radial gradient distribution of DNA, with higher concentrations at the particle's surface. 


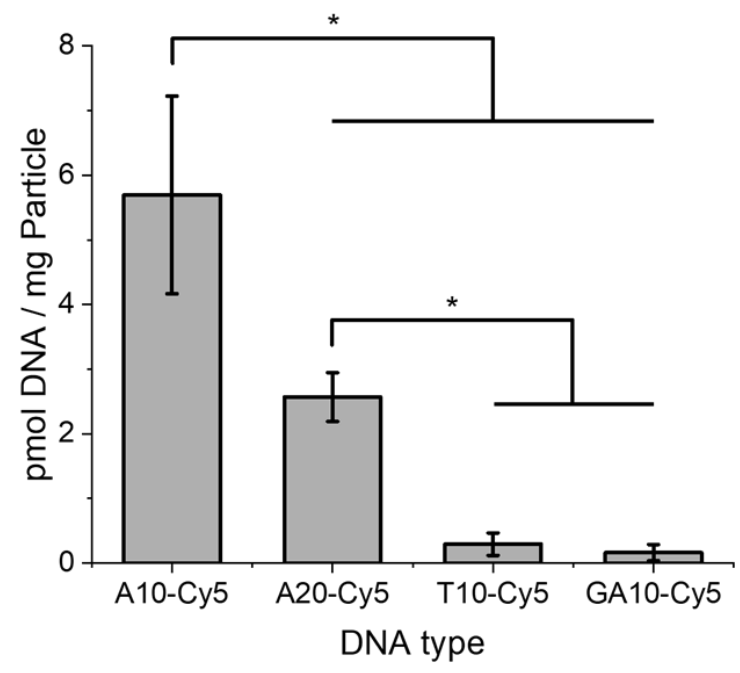

Figure S4-MP+oT microparticles were loaded with A10-Cy5 DNA, A20-Cy5 DNA, T10-Cy5 DNA, and GA10-Cy5 DNA. Loading was significantly greater for the A10-Cy5 DNA, but significant loading was also observed for A20-Cy5 DNA. The reduction in loading of A20-Cy5 is most likely due to the longer structure, which could potentially bind twice as many CNA oligomers as A10-Cy5 as well as experience hindered transport in the particle. There was negligible loading for the non-complementary T10-Cy5 DNA and the periodically mismatched GA10-Cy5 DNA. These results support the specificity of the CNA/DNA mediated loading and demonstrates the microparticles ability to load DNA of different sizes. 
a Calibration Curve for Temperature Dependent Release

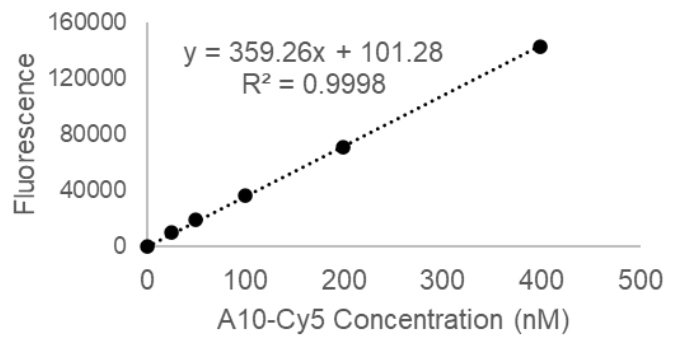

C Calibration Curve for Release at $\mathrm{pH} 7.3$

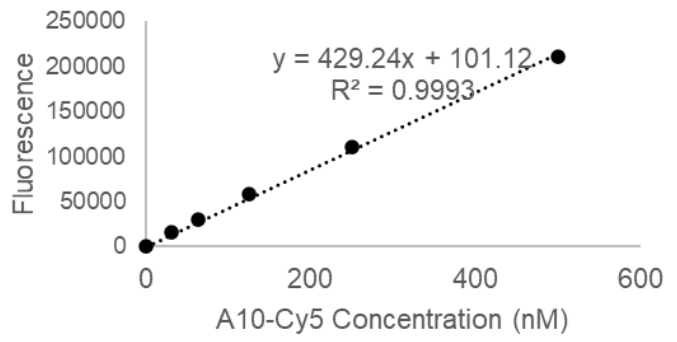

b Calibration Curve for Release at $\mathrm{pH} 8.5$

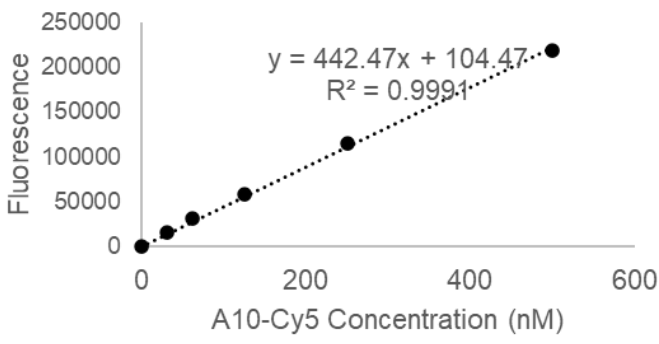

d Calibration Curve for Release at $\mathrm{pH} 5.8$

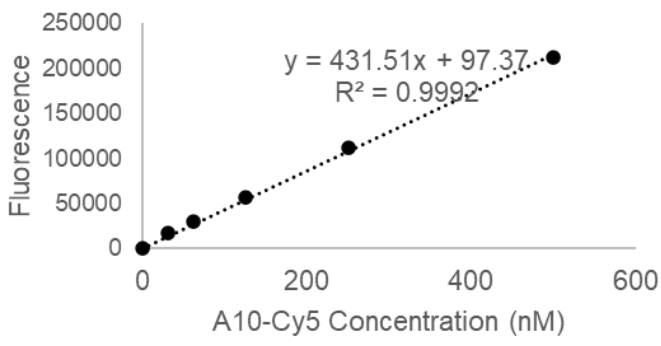

Figure S5 - Calibration curves to quantify the release of A10-Cy5 from microparticles under various conditions. (a) Calibration curve for long term, temperature dependent release. (b), (c), and (d) Calibration curves for $\mathrm{pH}$ dependent release. Calibration curves were made for each $\mathrm{pH}$ due to the possible dependence of Cy5 fluorescence on $\mathrm{pH}$.
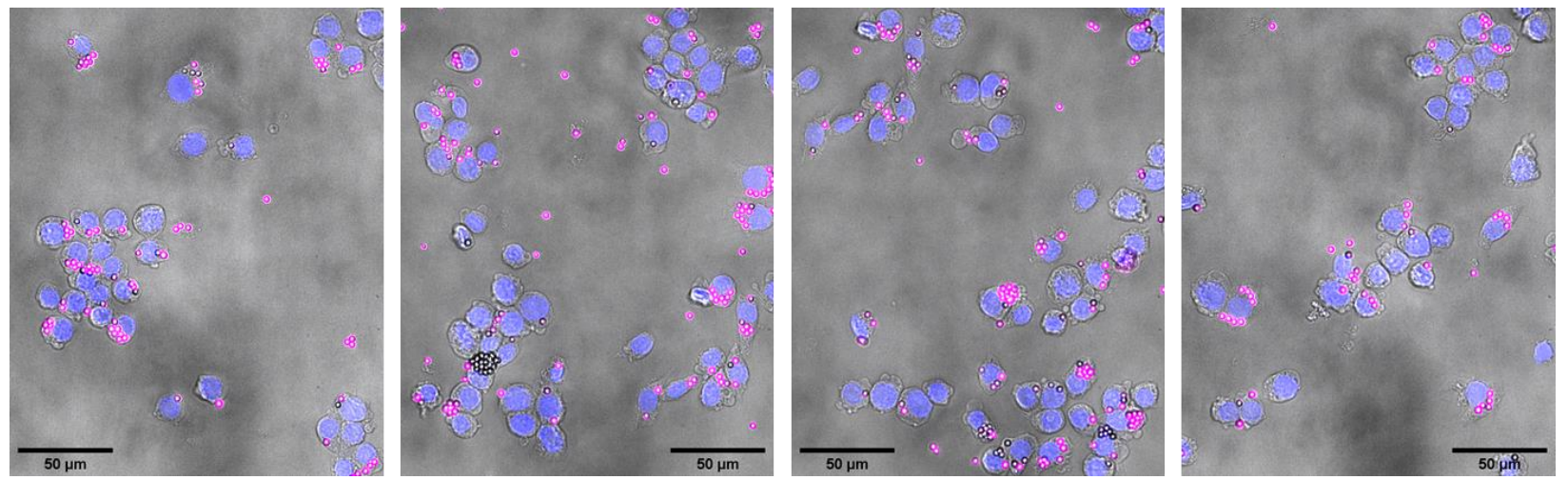

Figure S6 - More representative images of microparticle uptake in RAW 264.7 cells. (Blue: nuclei; Magenta: A10-cy5 loaded MP+oT) 


\section{References}

(1) Wang, C.; Zhang, X.; Podgórski, M.; Xi, W.; Shah, P.; Stansbury, J.; Bowman, C. N. Monodispersity/Narrow Polydispersity Cross-Linked Microparticles Prepared by Step-Growth Thiol-Michael Addition Dispersion Polymerizations. Macromolecules 2015, 48 (23), 8461-8470. https://doi.org/10.1021/acs.macromol.5b02146.

(2) Wang, C.; Podgórski, M.; N. Bowman, C. Monodisperse Functional Microspheres from StepGrowth "Click" Polymerizations: Preparation, Functionalization and Implementation. Materials Horizons 2014, 1 (5), 535-539. https://doi.org/10.1039/C4MH00082J.

(3) Anderson, A. J.; Peters, E. B.; Neumann, A.; Wagner, J.; Fairbanks, B.; Bryant, S. J.; Bowman, C. N. Cytocompatibility and Cellular Internalization of PEGylated "Clickable" Nucleic Acid Oligomers. Biomacromolecules 2018, 19 (7), 2535-2541. https://doi.org/10.1021/acs.biomac.8b00162.

(4) Han, X.; Domaille, D. W.; Fairbanks, B. D.; He, L.; Culver, H. R.; Zhang, X.; Cha, J. N.; Bowman, C. N. New Generation of Clickable Nucleic Acids: Synthesis and Active Hybridization with DNA. Biomacromolecules 2018, 19 (10), 4139-4146. https://doi.org/10.1021/acs.biomac.8b01164. 